\title{
Unified approach of coupled constitutive laws
}

\author{
F. Collin*, L. Laloui**, R. Charlier* \\ *Université de Liège, Institut de Génie Civil - bât. B52, chemin des Chevreuils n¹, \\ B-4000 Liège 1, Belgium - Email : Robert.Charlier@ULg.ac.be \\ **Soil Mechanics Laboratory - Ecole Polytechnique Fédérale de Lausanne, EPFL \\ CH-1015 Lausanne, Switzerland - E-mail: Lyesse.Laloui@epfl.ch
}

RÉSUMÉ : Plusieurs modèles de comportement des géomatériaux ont été publiés pour la prise en compte du couplage entre la mécanique et i) les écoulements fluides multiphasiques et la succion, ii) les transferts thermiques, et iii) plus récemment les modifications de concentration chimique. La plupart de ces modèles reposent sur des bases communes. Cellesci sont exposées puis particularisées aux 3 couplages considérés.

ABSTRACT: A number of geomaterials constitutive models have been proposed for the coupling of the mechanical behaviour with i) multi-phase fluid flow and suction level, ii) the thermal field changes and iii) more recently the chemical concentration evolution. Most of these models follow a common framework that will be first emphasised and then particularised for the 3 considered couplings.

MOTS-CLÉS : loi de comportement, THMC, chimio-thermo-hydro-mécanique

KEYWORDS: constitutive law, THMC, chemo-thermo-hydro-mechanics,

REGC - 9/2005. Multiphysics Geomechnics, pages 713 to 723 


\section{Introduction}

A number of constitutive models have been proposed during the last two decades to describe separately the behaviour of soils submitted to suction (unsaturated soil mechanics), to temperature (thermo-mechanics) as well as to chemistry (chemo-mechanics) loadings. Such solicitations are the main acting forces in the field of environmental geomechanics [VUL 02]. For unsaturated soil mechanics, the Barcelona Basic Model [ALO 90] was a starting point for a large number of researches. Among others Collin et al. [COL 02] proposed adaptations of the latter model for chalk material. A chemo-plasticity model was proposed by Hueckel [HUE 97] for clay. It was later reworked by Boukpeti et al. [BOU 03] and Liu et al [LIU 05]. Other contributions were proposed e.g. on rock weathering by Nova et al. [NOV 05]. Thermo-plastic models were developed by Hueckel and Borsetto [HUE 90], Laloui [LAL 93], Modaressi and Laloui [MOD 97]. All these contributions are complementary, and some interest was put recently on coupling between two such environmental loadings and mechanics, e.g. thermo-mechanics of unsaturated soils or chemo-mechanics of unsaturated soils. The question now arrives on how to experiment in the lab for such multi-coupled phenomena and how to build adequate constitutive frameworks.

This paper intends to present a unified approach to point out a general framework for expressing the constitutive modelling of soils in geo-environmental conditions. From the authors' point of view, this should help to analyse and to model different multi-physics phenomena, and to develop new multi-coupling approaches. We will first derive a generic form for all these constitutive models, and then consider the main effects of each of those geo-environmental loadings. In this paper, we only focus on mechanical constitutive aspects, neglecting the diffusion, conduction and other transport phenomena.

\section{Generic structure for constitutive models dedicated to multi-physics coupling.}

\subsection{Variables and notations}

In the following, $\varepsilon_{\mathrm{ij}}$ denotes the strain tensor and $\sigma_{\mathrm{ij}}$ the stress tensor. We will consider only one coupling, between the mechanical behaviour and one environmental phenomenon: temperature $(T)$, suction (s), or chemistry $(c)$, denoted generically $\gamma$, which is always a scalar.

For simplicity, the mechanical behaviour is described by the Cam-Clay concept. Elastoplasticity is considered, i.e. strain supports an additive decomposition into reversible - elastic and irreversible - plastic parts: 


$$
\dot{\varepsilon}_{i j}=\dot{\varepsilon}_{i j}^{e}+\dot{\varepsilon}_{i j}^{p}
$$

Strain variations are induced by either stress variation (denoted by $m$ exponent) or by a variation of the environmental variable $\gamma$ (denoted by $\gamma$ exponent). The strain rate writes:

$$
\begin{gathered}
\dot{\varepsilon}_{i j}=\dot{\varepsilon}_{i j}^{m}+\dot{\varepsilon}_{i j}^{\gamma} \\
\dot{\varepsilon}_{i j}^{m}=\dot{\varepsilon}_{i j}^{e, m}+\dot{\varepsilon}_{i j}^{p, m} \\
\dot{\varepsilon}_{i j}^{\gamma}=\dot{\varepsilon}_{i j}^{e, \gamma}+\dot{\varepsilon}_{i j}^{p, \gamma}
\end{gathered}
$$

Internal variables are the stress tensor, $\sigma_{\mathrm{ij}}$, the coupling variable, $\gamma$, and memory variables as the void ratio, $e$, and/or the plasticity threshold, like the preconsolidation pressure, $p_{0}$. The stresses and the coupling variable play the same role in the model elaboration. In order to simplify the writing of the time integration algorithms for the constitutive model, Vaunat et al. [VAU 00] suggest the use of generalized variables:

$$
\begin{gathered}
\underline{\sigma}^{*}=\left(\bar{\sigma}_{11}, \bar{\sigma}_{22}, \bar{\sigma}_{33}, \bar{\sigma}_{12}, \bar{\sigma}_{13}, \bar{\sigma}_{23}, \gamma\right)^{t} \\
\underline{\varepsilon}^{*}=\left(\varepsilon_{11}, \varepsilon_{22}, \varepsilon_{33}, \varepsilon_{12}, \varepsilon_{13}, \varepsilon_{23}, \varepsilon_{v}^{\gamma}\right)^{t}
\end{gathered}
$$

where $\varepsilon_{\mathrm{ij}}$ are the total strains and $\varepsilon_{\mathrm{v}}{ }^{\gamma}$ is the volumetric strain induced by the coupling variable rate. It is commonly considered that the coupling only induces volumetric strains and not deviatoric ones. Moreover, total strain rates are considered (and not only purely mechanical induced strains) as they are the basic input in time integration scheme in finite element codes.

\subsection{Elasticity}

The elasticity constitutive model for the mechanical part writes:

$$
\dot{\sigma}_{k l}=C_{k l i j}^{e} \dot{\varepsilon}_{i j}^{m, e}
$$

With the elasticity tensor $C_{k l i j}^{e}$ defined as:

$$
C_{k l i j}^{e}=2 G \delta_{i k} \delta_{j l}+\left[\frac{(1+e)}{3 \kappa} \sigma_{m m}-\frac{2}{3} G\right] \delta_{i j} \delta_{k l}
$$

where $\delta_{i j}$ is the Kronecker symbol, $G$ the shear modulus, $\kappa$ the volumetric elastic modulus (logarithmic law). The material parameters $G$ and $\kappa$ may depend on the coupling variable $\gamma$. The elastic strain induced by the coupling variable $\gamma$ rate is a purely volumetric one:

$$
\dot{\varepsilon}_{i j}^{e, \gamma}=K \dot{\gamma} \delta_{i j}
$$


It may be a non-linear relation, i.e. $K$ may depend on the stress and coupling variable states. This model indicates that changes related to chemistry, temperature, or suction may induce micro-structural changes, and, ions and water molecules movements. The elastic laws may rewrite:

$$
\begin{gathered}
\underline{\dot{\sigma}}=\underline{\underline{C}}^{e} \underline{\dot{\varepsilon}}^{e, m}=\underline{C}^{e} \cdot\left(\underline{\dot{\varepsilon}}^{e}-\dot{\varepsilon}_{v}^{e, \gamma} \frac{I}{3}\right)=\underline{C}^{e} \underline{\dot{\varepsilon}}^{e}-\chi \dot{\varepsilon}_{v}^{\gamma, e} \underline{I} \\
\dot{\gamma}=\frac{1}{K} \dot{\varepsilon}_{v}^{e, \gamma}
\end{gathered}
$$

with $\chi$ the volumetric elasticity modulus. Considering the generalized variables [3]:

$$
\underline{\dot{\sigma}}^{*}=\underline{\underline{C}}^{* e} \cdot \underline{\dot{\varepsilon}}^{* e}
$$

with the following elasticity matrix (3D problems):

$$
\stackrel{C^{* e}}{=}=\left[\begin{array}{ccccccc}
\chi+\frac{4}{3} G & \chi-\frac{2}{3} G & \chi-\frac{2}{3} G & 0 & 0 & 0 & -\chi \\
\chi-\frac{2}{3} G & \chi+\frac{4}{3} G & \chi-\frac{2}{3} G & 0 & 0 & 0 & -\chi \\
\chi-\frac{2}{3} G & \chi-\frac{2}{3} G & \chi+\frac{4}{3} G & 0 & 0 & 0 & -\chi \\
0 & 0 & 0 & 2 G & 0 & 0 & 0 \\
0 & 0 & 0 & 0 & 2 G & 0 & 0 \\
0 & 0 & 0 & 0 & 0 & 2 G & 0 \\
0 & 0 & 0 & 0 & 0 & 0 & 1 / K
\end{array}\right]
$$

\subsection{Plasticity threshold}

The yield surface has to take into account three types of mechanical behaviours:

- Nearly isotropic compression, characterised by a pre-consolidation pressure as plasticity threshold $p_{0}$;

- Deviatoric shear failure based on internal friction model and friction angle $\phi$ or $p$ - $q$ slope $M$;

- $\quad$ Traction strength $\sigma_{t}$, isotropic traction $p_{s}$ or cohesion $c$.

These three parameters $p_{0}, M$ and $\sigma_{t}$ may depend on the coupling variable $\gamma$.

A schematic view of such a yield surface is given in figure 1-a. In the $p$ - $q$ invariant plane, it is composed of an elliptic cap, a friction line and a traction cutoff. 
(a)

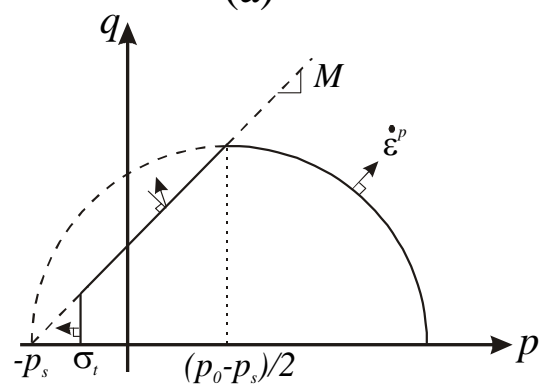

(b)

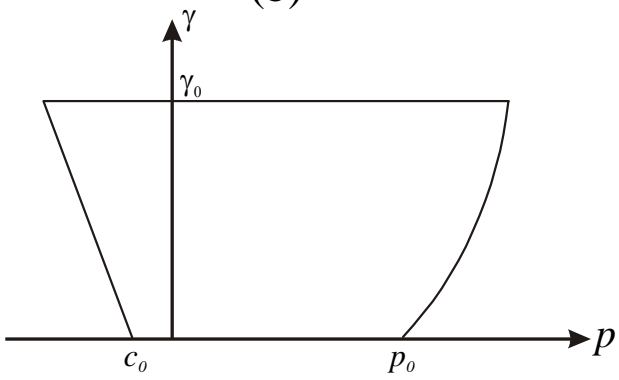

Figure 1: Yield surface for purely mechanical problems (a) and for Mechanical - environmental coupling (b).

This yield surface writes:

$$
\begin{aligned}
& f_{1}=q^{2}+M^{2}\left(p+p_{s}\right)\left(p-p_{0}\right)=0 ; p \geq\left(p_{0}-p_{s}\right) / 2 \\
& f_{2}=q-M\left(p+p_{s}\right)=0 ; \sigma_{\mathrm{t}}<p \leq\left(p_{0}-p_{s}\right) / 2 \\
& f_{3}=p+\sigma_{t}=0
\end{aligned}
$$

The slope $M$ may depend on the Lode angle, as proposed e.g. by Van Eekelen [EEK 80].

Additionally, a specific plasticity threshold may be written for the coupling variable:

$$
\gamma=\gamma_{0}
$$

The mechanical - environmental coupling is schematised for the yield surface in the figure 1-b, where the coupling between the environmental variable $\gamma$ and respectively the pre-consolidation pressure $p_{0}$, and the traction strength $\sigma_{t}$.

\subsection{Plastic strains}

The plastic strain rate derives from a flow potential which often differs from the yield surface (non-associative plasticity) in the stress plane (figure 1-a) but generally coincides with the yield surface in the coupling plane (figure 1-b).

Once again the coupling term is a purely volumetric one. With the generalized variables, this writes: 


$$
\underline{\dot{\varepsilon}}^{* p}=\underline{\dot{\varepsilon}}^{p, m}+\dot{\varepsilon}_{v}^{p, \gamma} \frac{\underline{I}}{3}=\dot{\lambda}^{p} \frac{\partial g}{\partial \underline{\sigma}^{*}}
$$

with :

$$
\frac{\partial g}{\partial \underline{\sigma}^{*}}=\left(\frac{\partial g}{\partial \sigma_{1}}+\frac{1}{3} \frac{\partial g}{\partial \gamma}, \frac{\partial g}{\partial \sigma_{2}}+\frac{1}{3} \frac{\partial g}{\partial \gamma}, \frac{\partial g}{\partial \sigma_{3}}+\frac{1}{3} \frac{\partial g}{\partial \gamma}, \frac{\partial g}{\partial \sigma_{4}}, \frac{\partial g}{\partial \sigma_{5}}, \frac{\partial g}{\partial \sigma_{6}}, \frac{\partial g}{\partial \gamma}\right)
$$

\subsection{Application to different coupling phenomena}

Generally speaking, it appears the following environmental dependences of the plastic material parameters, more precisely the yield surface parameters:

- The pre-consolidation pressure $p_{0}$ is highly dependent on the environmental phenomena;

- The friction angle at the critical state $\phi$ seems quite independent on the environmental phenomena;

- The cohesion $c$ and the traction strength $\sigma_{t}$ depend on the suction and on the chemistry (damage, weathering of cementation bridges). However the know-how on these aspects are not highly developed.

Following these observations, most papers have been devoted to the cap of the yield surface, i.e. the Cam-Clay equation $f_{1}$ (see Equation 10), and to the preconsolidation pressure environmental evolution. The hardening law will govern this evolution. It depends on two internal variables:

$$
p_{0}=\Phi\left(p_{0}^{*}, \gamma\right)
$$

where $p_{0}{ }^{*}$ is the $p_{0}$ value when the coupling variable $\gamma$ vanishes.

Classically, $p_{0}{ }^{*}$ evolves with the volumetric plastic strain, following the CamClay concept:

$$
\dot{p}_{0}^{*}=\frac{1+e}{\lambda-\kappa} p_{0}^{*} \dot{\varepsilon}_{v}^{p}
$$

Deriving equation [16] with respect to the time:

$$
\dot{p}_{0}=\frac{\partial \Phi}{\partial p_{0}^{*}} \dot{p}_{0}^{*}+\frac{\partial \Phi}{\partial \gamma} \dot{\gamma}=\frac{\partial \Phi}{\partial p_{0}^{*}} \frac{1+e}{\lambda-\kappa} p_{0}^{*} \dot{\varepsilon}_{v}^{p}+\frac{\partial \Phi}{\partial \gamma} \dot{\gamma}
$$

The preconsolidation pressure rate depends on the plastic volumetric strain $\varepsilon_{v}^{p}$ and on the coupling variable $\gamma$. If one considers a plastic loading where the stresses do not vary, following the consistency condition: 


$$
f=\frac{\partial f}{\partial \sigma_{i j}} \dot{\sigma}_{i j}+\frac{\partial f}{\partial p_{0}} \dot{p}_{0}=0
$$

and

$$
\dot{p}_{0}=0
$$

Then the plastic strain rate induced by a purely environmental loading writes:

$$
\dot{\varepsilon}_{v}^{p}=\frac{-\frac{\partial \Phi}{\partial \gamma} \dot{\gamma}}{\frac{\partial \Phi}{\partial p_{0}^{*}} \frac{1+e}{\lambda-\kappa} p_{0}^{*}}
$$

\section{Model application to suction, thermal or chemical coupling}

In the following development, $s$ denotes the suction in the hydraulic partly saturated coupling, $T$ the temperature for the heat coupled model and $c$ a measure of the concentration for the chemical coupled model. The same letters will be used as indices for denoting the variables, in place of the generic notation $\gamma$. The considered models here are based, respectively,

- for the hydraulic partly saturated coupling, on the Barcelona Basic Model [ALO 90]

- $\quad$ for the chemical coupling on the Hueckel model [HUE 97]

- $\quad$ for the thermal coupling on the Laloui and Cekerevac model [LAL 03].

\subsection{Elastic strains}

In this section we describe the reversible strain induced by the environmental load. Such elastic strain may be expressed simply as proportional to the variation of the considered load (as for the chemical load). However, for suction and temperature effects, the reversible strain is no more linear and complementary dependency laws are needed.

\subsubsection{Suction coupling}

A suction increase induces a strain contraction, which is often described with a logarithmic incremental law (figure 2a):

$$
\dot{\varepsilon}_{v}^{s, e}=\frac{1}{3} \frac{\kappa_{s}}{(1+e)} \frac{\dot{s}}{\left(s+p_{a t}\right)}
$$


where $\kappa_{s}$ is a compressibility constant parameter and $p_{a t}$ denotes the atmospheric pressure.

\subsubsection{Chemical coupling}

A chemical concentration increase induces a volumetric dilatation (figure 2b):

$$
\varepsilon_{v}^{c, e}=F_{0} \exp \left[\beta_{0}(1-c+\ln c)\right]
$$

where $F_{0}$ denotes the maximal strain, for a concentration equal to unity. $\beta_{0}$ is the curve slope for half of the maximum strain $F_{0} / 2$. An incremental formulation follows:

$$
\begin{aligned}
& \dot{\varepsilon}_{i j}^{c, e}=-\frac{1}{3} \beta \dot{c} \delta_{i j} \\
& \beta=-F_{0} \beta_{0} \exp \left[\beta_{0}(1-c+\ln c)\right]\left(\frac{1}{c}-1\right)
\end{aligned}
$$

(a)

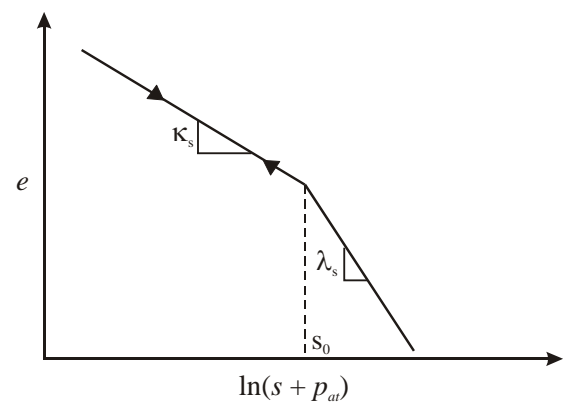

(b)

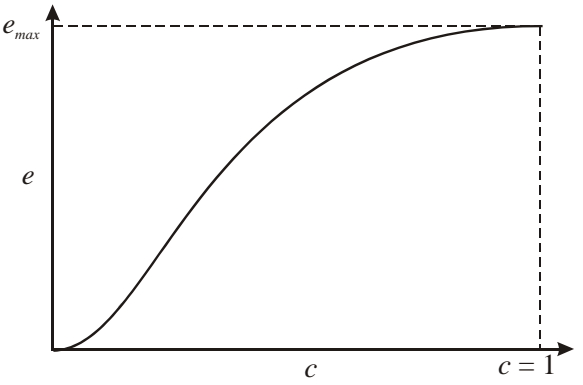

Figure 2 : Elastic behaviour induced by a change of, (a) suction, (b) concentration.

\subsubsection{Thermal coupling}

The temperature increase induces a non linear elastic dilatation:

$$
\varepsilon_{v}^{T, e}=\beta_{s}\left(T-T_{0}\right)
$$

where $\varepsilon_{v}^{T, e}$ is the volumetric thermo-elastic strain, $\beta_{s}$ the volumetric thermal dilation coefficient and $T-T_{0}$ the thermal variation. The non-linear dependency of the thermal dilation coefficient is introduced with respect to the consolidation stress and temperature:

$$
\beta_{\mathrm{s}}=\left(\beta_{\mathrm{s} 0}+\zeta\left(T-T_{0}\right)\right) \xi
$$


Where $\beta_{\mathrm{s} 0}$ is the volumetric thermal expansion coefficient at the reference temperature, $\xi$ a parameter function of effective mean pressure and $\zeta$ a (negative) material parameter inducing a non-linear dependency to the temperature level. The dilatation coefficient is then decreasing when the temperature increases. This formulation enables the thermo-elastic strain rate to be more pronounced for higher values of over-consolidation ratio and temperature.

\subsection{Plasticity yield surface}

The yield surface depends on 3 parameters: the pre-consolidation pressure, $p_{0}$, the friction angle, $\phi$, and the cohesion, $c$.

\subsubsection{Suction coupling}

In the Barcelona Basic Model, the preconsolidation pressure is governed by:

$$
p_{0}=p_{c}\left(\frac{p_{0}^{*}}{p_{c}}\right)^{\frac{\lambda(0)-\kappa}{\lambda(s)-\kappa}}
$$

Basically, drying a soil increases its strength. Other shape may be used (e.g. for chalk, see [COL 03]). The friction angle is generally considered as quite independent from the suction. In the Barcelona Basic Model, the cohesion model is linear:

$$
c=c_{0}+k \cdot S
$$

\subsubsection{Chemical coupling}

In the Hueckel Model [HUE 97], the preconsolidation pressure is governed by:

$$
p_{0}=p_{0}^{*} S(c) ; \quad S(c)=\exp (-a c)
$$

The strength is reduced by an increase in chemical concentration. The friction angle is generally considered as quite independent from the chemistry. Few exist about the cohesion. Cohesion could be weathered by concentration increase [NOV 05].

\subsubsection{Thermal coupling}

In the Laloui and Cekerevac Model [LAL 03], the apparent preconsolidation pressure decreases with the temperature increase:

$$
p_{0}(T)=p_{0}^{*}\left(T_{0}\right)\left\{1-\lambda \log \left[T / T_{0}\right]\right\}
$$

$\lambda$ being a material parameters [LAL 05]. The friction angle may be considered as quite independent from the temperature [CEK 04]. The plastic thermal effect induces a more ductile behaviour. 


\section{Conclusion}

It has been shown that many constitutive models for mechanical coupling with I) fluid multiphase flow, ii) thermal transfers, and iii) chemical concentration variations, may be considered as build on a common framework. Rewriting these equation within this framework allows first to emphasize the basic modelling choices that have been done, and secondly to elaborate new and multiphysics coupled models. Moreover such common framework could be a guide for some experimental programs, as it allows asking questions on the physical answer of materials under coupled loading.

\section{Acknowledgment}

The authors are grateful for support of their researches to the ESF under COST 351 WATMOVE action, and to the Belgian Fonds National de la Recherche Scientifique.

\section{References}

[ALO 90] Alonso E.E., Gens A., Josa A., A constitutive model for partially saturated soils. Géotechnique 40(3), pp 405-430,1990.

[BOU 03] Boukpeti N., Charlier R., Hueckel T., Modelling contamination of clays, VII International conference on Computational Plasticity, Owen DRJ, Onate E, Suarez B (eds), Barcelona, 2003, 19 p.

[COL 02] Collin F., Cui Y.J., Schroeder C., Charlier R., Mechanical behaviour of Lixhe chalk partly saturated by oil and water: experiment and modeling. Numerical and Analytical Methods in Geomechanics, 26, 2002, pp. 897-924.

[COL 03] Collin F. Couplages thermo-hydro-mécaniques dans les sols et les roches tenders partiellement saturés. Doctoral Thesis, University of Liège, 2003.

[CEK 04] Cekerevac C., Laloui L., "Experimental study of the thermal effects on the mechanical behaviour of a clay". International Journal of Numerical and Analytical Methods in Geomechanics, 28, 2004, pp 209-228.

[HUE 97] Hueckel T., Chemo-plasticity of clays subjected to stress and flow of a single contaminant. International Journal for Numerical and Analytical Methods in Geomechanics, 21, 1997, pp 43-72.

[HUE 90] Hueckel T., Borsetto M., Thermoplasticity of saturated soils and shales: Constitutive equations. Journal of Geotechnical Engineering, 116(2), 1990, pp 17651777.

[LAL 93] Laloui L. Modélisation du comportement thermo-hydro-mécanique des milieux poreux anélastiques. Doctoral Thesis, Ecole Centrale Paris, 1993. 
[LAL 03] Laloui L., Cekerevac C., Thermo-plasticity of clays: an isotropic yield mechanism. Computers and Geotechnics, 30(8), 2003, pp 649-660.

[LAL 05] Laloui L., Cekerevac C., François B., Constitutive modelling of the thermo-plastic behaviour of soils. Revue Européenne de Génie Civil, 2005.

[LIU 05] Liu Z., Boukpeti N., Li X., Collin F., Radu J.P., Hueckel T., Charlier R.. Modelling chemo-hydro-mechanical behaviour of unsaturated clays : a feasibility study. Accepted for publication in International Journal for Numerical and Analytical Methods in Geomechanics, 2005.

[MOD 97] Modaressi H., Laloui L., Thermo-viscoplastic model for clays. International Journal of Numerical and Analytical Methods in Geomechanics, 21, 1997, pp 313-335.

[NOV 05] Nova R., Parma M., An instructive chemo-mechanical model for bonded geomaterials. Revue Européenne de Génie Civil, 2005.

[EEK 80] Van Eekelen H.A.M., Isotropic yield surfaces in three dimensions for use in soil mechanics. International Journal for Numerical and Analytical Methods in Geomechanics, 4, 1980, pp 98-101.

[VAU 00] Vaunat J., Cante J.C., Ledesma A., Gens A., A stress point algorithm for an elastoplastic model in unsaturated soils. International Journal of Plasticity, 16, 2000, pp. 121-141.

[VUL 02] Vulliet L., Laloui L., Schrefler B, Environmental geomechanics. EPFL-Press. 423 pages, 2002. 